\title{
ARGUMENT ESTIMATES OF MEROMORPHICALLY MULTIVALENT FUNCTIONS
}

\section{$\operatorname{AUTHOR}(\mathrm{S})$ :}

Cho, Nak Eun; Owa, Shigeyoshi

\section{CITATION:}

Cho, Nak Eun ...[et al]. ARGUMENT ESTIMATES OF MEROMORPHICALLY MULTIVALENT FUNCTIONS. 数理解析研究所講究録 1999, 1112: 1-12

ISSUE DATE:

1999-09

URL:

http://hdl.handle.net/2433/63368

RIGHT: 


\title{
ARGUMENT ESTIMATES OF MEROMORPHICALLY MULTIVALENT FUNCTIONS
}

\author{
*NAK Eun Cho AND **Shigeyoshi OWA
}

\begin{abstract}
The object of the present paper is to obtain some argument properties of meromorphically multivalent functions in the punctured open unit disk. We also derive the integral preserving properties in a sector.
\end{abstract}

\section{Introduction}

Let $\mathcal{U}=\{z \in \mathbb{C}:|z|<1\}$. For $f$ and $g$ which are analytic in $\mathcal{U}$, we say that $f$ is subordinate to $g$, written $f \prec g$ or $f(z) \prec g(z)$, if there exists a Schwarz function $w$ in $\mathcal{U}$ such that $f(z)=g(w(z))$.

Let $\Sigma_{p}$ denote the class of all meromorphic functions of the form

$$
f(z)=\frac{1}{z^{p}}+\frac{a_{0}}{z^{p-1}}+\ldots+a_{k+p-1} z^{k}+\ldots(p \in \mathbb{N}=\{1,2, \cdots\})
$$

which are analytic in the annulus $\mathcal{D}=\{z: 0<|z|<1\}$. We denote by $\Sigma_{p}^{*}(\beta)$ the subclass of $\Sigma_{p}$ consisting of all functions which is meromorphically starlike of order $\beta$ in $\mathcal{U}$.

The Hadamard product or convolution of two functions $f$ and $g$ in $\Sigma_{p}$ will be denoted by $f * g$.

Let

$$
D^{n+p-1} f(z)=\frac{1}{z^{p}(1-z)^{n+p}} * f(z)(z \in \mathcal{D})
$$

or, equivalently,

1991 Mathematics Subject Classification: 30C45.

Key words and phrases. meromorphically starlike, differential operator, argument estimates, integral operator. 


$$
\begin{aligned}
D^{n+p-1} f(z)= & \frac{1}{z^{p}}\left(\frac{z^{n+2 p-1} f(z)}{(n+p-1) !}\right)^{(n+p-1)} \\
= & \frac{1}{z^{p}}+(n+p) a_{0} \frac{1}{z^{p-1}}+\frac{(n+p+1)(n+p)}{2 !} a_{1} \frac{1}{z^{p-2}}+\ldots \\
& \ldots+\frac{(n+k+2 p-1) \ldots(n+p)}{(k+p) !} a_{k+p-1} z^{k}+\ldots \quad(z \in \mathcal{D}),
\end{aligned}
$$

where $n$ is any integer greater than $-p$.

For various interesting developments involving the operators $D^{n+p-1}$ for functions belonging to $\Sigma_{p}$, the reader may be refereed to the recent works of author[1], Uralegaddi and Path[7], and others[8,9].

Let

$$
\Sigma_{p}^{*}[n ; A, B]=\left\{f \in \Sigma_{p}:-\frac{z\left(D^{n+p-1} f(z)\right)^{\prime}(z)}{D^{n+p-1} f(z)} \prec p \frac{1+A z}{1+B z}, z \in \mathcal{U}\right\}
$$

where $-1 \leq B<A \leq 1$. In particular, we note that $\Sigma_{p}^{*}[-p+1 ; 1,-1]$ is the well known class of meromorphically $p$-valent starlike functions. From (1.2), we observe[6] that a function $f$ is in $\Sigma_{p}^{*}[n ; A, B]$ if and only if

$$
\left|\frac{z\left(D^{n+p-1} f(z)\right)^{\prime}(z)}{D^{n+p-1} f(z)}+\frac{p(1-A B)}{1-B^{2}}\right|<\frac{p(A-B)}{1-B^{2}}(-1<B<A \leq 1 ; z \in \mathcal{U})
$$

The object of the present paper is to give some argument estimates of meromorphically multivalent functions belonging to $\Sigma_{p}$ and the integral preserving properties in connection with the differential operators $D^{n+p-1}$ defined by (1.1).

\section{Main results}

To establish our main results, we need the following lemmas.

Lemma 2.1 [2]. Let $h$ be convex univalent in $\mathcal{U}$ with $h(0)=1$ and $\operatorname{Re}(\beta h(z)+$ $\gamma)>0(\beta, \gamma \in \mathbb{C})$. If $q$ is analytic in $\mathcal{U}$ with $q(0)=1$, then

$$
q(z)+\frac{z q^{\prime}(z)}{\beta q(z)+\gamma} \prec h(z) \quad(z \in \mathcal{U})
$$

implies 


\section{ARGUMENT ESTIMATES}

$$
q(z) \prec h(z) \quad(z \in \mathcal{U})
$$

Lemma 2.2 [4]. Let $h$ be convex univalent in $\mathcal{U}$ and $\lambda(z)$ be analytic in $\mathcal{U}$ with $\operatorname{Re} \lambda(z) \geq 0$. If $q$ is analytic in $\mathcal{U}$ and $q(0)=h(0)$, then

$$
q(z)+\lambda(z) z q^{\prime}(z) \prec h(z) \quad(z \in \mathcal{U})
$$

implies

$$
q(z) \prec h(z) \quad(z \in \mathcal{U})
$$

Lemma 2.3 [5]. Let $q$ be analytic in $\mathcal{U}$ with $q(0)=1$ and $q(z) \neq 0$ in $\mathcal{U}$. Suppose that there exists a point $z_{0} \in \mathcal{U}$ such that

$$
|\arg q(z)|<\frac{\pi}{2} \alpha \text { for }|z|<\left|z_{0}\right|
$$

and

$$
\left|\arg q\left(z_{0}\right)\right|=\frac{\pi}{2} \alpha \quad(0<\alpha \leq 1)
$$

Then we have

$$
\frac{z_{0} q^{\prime}\left(z_{0}\right)}{q\left(z_{0}\right)}=i k \alpha
$$

where

$$
\begin{gathered}
k \geq \frac{1}{2}\left(a+\frac{1}{a}\right) \text { when } \arg q\left(z_{0}\right)=\frac{\pi}{2} \alpha \\
k \leq-\frac{1}{2}\left(a+\frac{1}{a}\right) \text { when } \arg q\left(z_{0}\right)=-\frac{\pi}{2} \alpha
\end{gathered}
$$

and

$$
q\left(z_{0}\right)^{\frac{1}{\alpha}}= \pm i a(a>0)
$$

At first, with the help of Lemma 2.1, we obtain the following

Proposition 2.1. Let $h$ be convex univalent in $\mathcal{U}$ with $h(0)=1$ and $\operatorname{Re} h$ be bounded in $\mathcal{U}$. If $f \in \Sigma_{p}$ satisfies the condition

$$
-\frac{z\left(D^{n+p} f(z)\right)^{\prime}}{p D^{n+p} f(z)} \prec h(z) \quad(z \in \mathcal{U}),
$$


then

$$
-\frac{z\left(D^{n+p-1} f(z)\right)^{\prime}}{p D^{n+p-1} f(z)} \prec h(z) \quad(z \in \mathcal{U})
$$

for $\max _{\boldsymbol{z} \in \mathcal{U}} \operatorname{Re} h(z)<\frac{n+2 p}{p}$ (provided $D^{n+p-1} f(z) \neq 0$ in $\mathcal{U}$ ).

Proof. Let

$$
q(z)=-\frac{z\left(D^{n+p-i} f(z)\right)^{\prime}}{p D^{n+p-1} f(z)}
$$

By using the equation

$$
z\left(D^{n+p-1} f(z)\right)^{\prime}=(n+p) D^{n+p} f(z)-(n+2 p) D^{n+p-1} f(z),
$$

we get

$$
q(z)-\frac{n+2 p}{p}=-\frac{(n+p) D^{n+1} f(z)}{p D^{n+p-1} f(z)}
$$

Taking logarithemic derivatives in both sides of (2.8) and multiplying by $z$, we have

$$
\frac{z q^{\prime}(z)}{-p q(z)+n+2 p}+q(z)=-\frac{z\left(D^{n+p} f(z)\right)^{\prime}}{p D^{n+p} f(z)} \prec h(z) \quad(z \in U) .
$$

From Lemma 2.1 , it follows that $q(z) \prec h(z)$ for $\operatorname{Re}\left(-h(z)+\frac{n+2 p}{p}\right)>0(z \in \mathcal{U})$, which means

$$
-\frac{z\left(D^{n+p-1} f(z)\right)^{\prime}}{p D^{n+p-1} f(z)} \prec h(z) \quad(z \in \mathcal{U})
$$

for $\max _{z \in \mathcal{U}} \operatorname{Re} h(z)<\frac{n+2 p}{p}$.

Proposition 2.2. Let $h$ be convex univalent in $\mathcal{U}$ with $h(0)=1$ and $\operatorname{Re} h$ be bounded in $\mathcal{U}$. Let $F$ be the integral operator defined by

$$
F(z)=\frac{c}{z^{c+p}} \int_{0}^{z} t^{c+p-1} f(t) d t \quad(c>0)
$$

If $f \in \Sigma_{p}$ satisfies the condition

$$
-\frac{z\left(D^{n+p-1} f(z)\right)^{\prime}}{p D^{n+p-1} f(z)} \prec h(z) \quad(z \in \mathcal{U})
$$

then

$$
-\frac{z\left(D^{n+p-1} F(z)\right)^{\prime}}{p D^{n+p-1} F(z)} \prec h(z) \quad(z \in \mathcal{U})
$$

for $\max _{z \in \mathcal{U}} \operatorname{Re} h(z)<\frac{c+p}{p}$ (provided $D^{n+p-1} F(z) \neq 0$ in $\mathcal{U}$ ). 
Proof. From (2.9), we have

$$
z\left(D^{n+p-1} F(z)\right)^{\prime}=c D^{n+p-1} f(z)-(c+p) D^{n+p-1} F(z) .
$$

Let

$$
p(z)=-\frac{z\left(D^{n+p-1} F(z)\right)^{\prime}}{p D^{n+p-1} F(z)}
$$

Then, by using (2.10), we get

$$
q(z)-(c+p)=-c \frac{D^{n+p-1} f(z)}{D^{n+p-1} F(z)} .
$$

Taking logarithemic derivatives in both sides of (2.11) and multiplying by $z$, we have

$$
\frac{z q^{\prime}(z)}{-p q(z)+(c+p)}+q(z)=-\frac{z\left(D^{n+p-1} f(z)\right)^{\prime}}{p D^{n+p-1} f(z)} \prec h(z) \quad(z \in \mathcal{U}) .
$$

Therefore, by Lemma 2.1, we have

$$
-\frac{z\left(D^{n+p-1} F(z)\right)^{\prime}}{p D^{n+p-1} F(z)} \prec h(z) \quad(z \in \mathcal{U})
$$

for $\max _{z \in \mathcal{U}} \operatorname{Re} h(z)<\frac{c+p}{p}$ (provided $D^{n+p-1} F(z) \neq 0$ in $\mathcal{U}$ ).

Remark. Taking $p=1$ and $h(z)=\frac{1+z}{1-z}$ in Proposition 2.1 and Proposition 2.2 , we have the results obtained by Ganigi and Uralegaddi[3].

Applying Lemma 2.2, Lemma 2.3 and Proposition 2.1, we now derive

Theorem 2.1. Let $f \in \Sigma_{p}$. Choose an integer $n$ such that

$$
n \geq \frac{p(1+A)}{1+B}-2 p
$$

where $-1<B<A \leq 1$ and $p \in \mathbb{N}$. If

$$
\left|\arg \left(-\frac{z\left(D^{n+p} f(z)\right)^{\prime}}{D^{n+p} g(z)}-\gamma\right)\right|<\frac{\pi}{2} \delta(0 \leq \gamma<p ; 0<\delta \leq 1)
$$

for some $g \in \Sigma_{p}^{*}[n+1 ; A, B]$, then

$$
\left|\arg \left(-\frac{z\left(D^{n+p-1} f(z)\right)^{\prime}}{D^{n+p-1} g(z)}-\gamma\right)\right|<\frac{\pi}{2} \alpha,
$$

where $\alpha(0<\alpha \leq 1)$ is the solution of the equation 
N. E. CHO AND S. OWA

$$
\delta=\alpha+\frac{2}{\pi} \tan ^{-1}\left(\frac{\alpha \sin \frac{\pi}{2}(1-t(A, B))}{\frac{(n+2 p)(1-B)+A-1}{1-B}+\alpha \cos \frac{\pi}{2}(1-t(A, B))}\right)
$$

when

$$
t(A, B)=\frac{2}{\pi} \sin ^{-1}\left(\frac{p(A-B)}{(n+2 p)\left(1-B^{2}\right)-p(1-A B)}\right) .
$$

Proof. Let

$$
q(z)=-\frac{1}{p-\gamma}\left(\frac{z\left(D^{n+p-1} f(z)\right)^{\prime}}{D^{n+p-1} g(z)}+\gamma\right)
$$

By (2.7), we have

$$
\begin{aligned}
& (p-\gamma) z q^{\prime}(z) D^{n+p-1} g(z)+(1-\gamma) q(z) z\left(D^{n+p-1} g(z)\right)^{\prime} \\
& -(n+2 p) z\left(D^{n+p-1} f(z)\right)^{\prime}=-(n+p) z\left(D^{n+p} f(z)\right)^{\prime}-\gamma z\left(D^{n+p-1} g(z)\right)^{\prime}(z)
\end{aligned}
$$

Dividing (2.14) by $D^{n+p-1} g(z)$ and simplifying, we get

$$
q(z)+\frac{z q^{\prime}(z)}{-r(z)+n+2 p}=-\frac{1}{p-\gamma}\left(\frac{z\left(D^{n+p} f(z)\right)^{\prime}}{D^{n+p} g(z)}+\gamma\right)
$$

where

$$
r(z)=-\frac{z\left(D^{n+p-1} g(z)\right)^{\prime}}{D^{n+p-1} g(z)} .
$$

Since $g \in \Sigma_{p}^{*}[n+1 ; A, B]$, from Proposition 2.1, we have

$$
r(z) \prec p \frac{1+A z}{1+B z} .
$$

Using (1.3), we have

$$
-r(z)+n+2 p=\rho e^{i \frac{\pi}{2}} \phi
$$

where

$$
\left\{\begin{array}{l}
\frac{(n+2 p)(1+B)-(1+A)}{1+B}<\rho<\frac{(n+2 p)(1-B)+A-1}{1-B} \\
-t(A, B)<\phi<t(A, B)
\end{array}\right.
$$




\section{ARGUMENT ESTIMATES}

when $t(A, B)$ is given by (2.13). Let $h$ be a function which maps $\mathcal{U}$ onto the angular domain $\left\{w:|\arg w|<\frac{\pi}{2} \delta\right\}$ with $h(0)=1$. Applying Lemma 2.2 for this $h$ with $\lambda(z)=\frac{1}{-r(z)+n+2 p}$, we see that $\operatorname{Re} q(z)>0$ in $\mathcal{U}$ and hence $q(z) \neq 0$ in $\mathcal{U}$.

If there exists a point $z_{0} \in \mathcal{U}$ such that the conditions (2.1) and (2.2) are satisfied, then(by Lemma 2.3) we obtain (2.3) under the restrictions (2.4), (2.5) and (2.6).

At first, suppose that $q\left(z_{0}\right)^{\frac{1}{\alpha}}=i a(a>0)$. Then we obtain

$$
\begin{aligned}
& \arg \left[-\frac{1}{p-\gamma}\left(\frac{z_{0}\left(D^{n+p} f\left(z_{0}\right)^{\prime}\right.}{D^{n+p} g\left(z_{0}\right)}+\gamma\right)\right]=\arg \left(q\left(z_{0}\right)+\frac{z_{0} q^{\prime}\left(z_{0}\right)}{-r\left(z_{0}\right)+n+2 p}\right) \\
& =\frac{\pi}{2} \alpha+\arg \left(1+i \alpha k\left(\rho e^{i \frac{\pi}{2} \phi}\right)^{-1}\right) \\
& =\frac{\pi}{2} \alpha+\tan ^{-1}\left(\frac{\eta k \sin \frac{\pi}{2}(1-\phi)}{\rho+\alpha k \cos \frac{\pi}{2}(1-\phi)}\right) \\
& \geq \frac{\pi}{2} \alpha+\tan ^{-1}\left(\frac{\alpha \sin \frac{\pi}{2}(1-t(A, B))}{\frac{(n+2 p)(1-B)+A-1}{1-B}+\alpha \cos \frac{\pi}{2}(1-t(A, B))}\right) \\
& =\frac{\pi}{2} \delta
\end{aligned}
$$

where $\delta$ and $t(A, B)$ are given by (2.12) and (2.13), respectively. This is a contradiction to the assumption of our theorem.

Next, suppose that $p\left(z_{0}\right)^{\frac{1}{\alpha}}=-i a(a>0)$. Applying the same method as the above, we have

$$
\begin{aligned}
& \arg \left[-\frac{1}{p-\gamma}\left(\frac{z_{0}\left(D^{n+p} f\left(z_{0}\right)\right)^{\prime}}{D^{n+p} g\left(z_{0}\right)}+\gamma\right)\right] \\
& \leq-\frac{\pi}{2} \alpha-\tan ^{-1}\left(\frac{\alpha \sin \frac{\pi}{2}(1-t(A, B))}{\frac{(n+2 p)(1-B)+A-1}{1-B}+\alpha \cos \frac{\pi}{2}(1-t(A, B))}\right) \\
& =-\frac{\pi}{2} \delta
\end{aligned}
$$

where $\delta$ and $t(A, B)$ are given by (2.12) and (2.13), respectively, which contradicts the assumption. Therefore we complete the proof of our theorem.

Letting $A=1, B=0$ and $\delta=1$ in Theorem 2.1, we have

Corollary 2.1. Let $f \in \Sigma$. If

$$
-\operatorname{Re}\left\{\frac{z\left(D^{n+p} f(z)\right)^{\prime}}{D^{n+p} g(z)}\right\}>\gamma(0 \leq \gamma<p)
$$


N. E. CHO AND S. OWA

for some $g \in \Sigma_{p}$ satisfying the condition

$$
\left|\frac{z\left(D^{n+p} g(z)\right)^{\prime}}{D^{n+p} g(z)}+p\right|<p
$$

then

$$
-\operatorname{Re}\left\{\frac{z\left(D^{n+p-1} f(z)\right)^{\prime}}{D^{n+p-1} g(z)}\right\}>\gamma
$$

Taking $A=1, B=0$ and $g(z)=\frac{1}{z^{p}}$ in Theorem 2.1, we have

Corollary 2.2. Let $f \in \Sigma_{p}$. If

$$
\left|\arg \left[-z^{p+1}\left(D^{n+p} f(z)\right)^{\prime}-\gamma\right]\right|<\frac{\pi}{2} \delta(0 \leq \gamma<p ; 0<\delta \leq 1)
$$

then

$$
\left|\arg \left[-z^{p+1}\left(D^{n+p-1} f(z)\right)^{\prime}-\gamma\right]\right|<\frac{\pi}{2} \delta
$$

Making $n=0, p=1$ and $\delta=1$ in Corollary 2.2, we have

Corollary 2.3. Let $f \in \Sigma_{1}$. If

$$
-\operatorname{Re}\left\{z^{2}\left(z f^{\prime \prime}(z)+3 f^{\prime}(z)\right)\right\}>\gamma(0 \leq \gamma<1)
$$

then

$$
-\operatorname{Re}\left\{z^{2} f^{\prime}(z)\right\}>\gamma
$$

By the same techniques as in the proof of Theorem 2.1, we obtain

Theorem 2.2. Let $f \in \Sigma$. Choose an integer $n$ such that

$$
n \geq \frac{p(1+A)}{1+B}-2 p
$$

where $-1<B<A \leq 1$ and $p \in \mathbb{N}$. If

$$
\left|\arg \left(\frac{z\left(D^{n+p} f(z)\right)^{\prime}}{\left(D^{n+p} g(z)\right)}+\gamma\right)\right|<\frac{\pi}{2} \delta(\gamma>p, 0<\delta \leq 1)
$$




\section{ARGUMENT ESTIMATES}

for some $g \in \Sigma_{p}^{*}[n+1 ; A, B]$, then

$$
\left|\arg \left(\frac{z\left(D^{n+p-1} f(z)\right)^{\prime}}{D^{n+p-1} g(z)}+\gamma\right)\right|<\frac{\pi}{2} \alpha,
$$

where $\alpha(0<\alpha \leq 1)$ is the solution of the equation given by (2.12).

Next, we prove

Theorem 2.3. Let $f \in \Sigma_{p}$ and choose a positive number $c$ such that

$$
c \geq \frac{1+A}{1+B}-p
$$

where $-1<B<A \leq 1$ and $p \in \mathbb{N}$. If

$$
\left|\arg \left(-\frac{z\left(D^{n+p-1} f(z)\right)^{\prime}}{D^{n+p-1} g(z)}-\gamma\right)\right|<\frac{\pi}{2} \delta(0 \leq \gamma<p ; 0<\delta \leq 1)
$$

for some $g \in \Sigma_{p}^{*}[n ; A, B]$, then

$$
\left|\arg \left(-\frac{z\left(D^{n+p-1} F(z)\right)^{\prime}}{D^{n+p-1} G(z)}-\gamma\right)\right|<\frac{\pi}{2} \alpha
$$

where $F$ is the integral operator given by (2.9),

$$
G(z)=\frac{c}{z^{c+p}} \int_{0}^{z} t^{c+p-1} g(t) d t, \quad(c>0),
$$

and $\alpha(0<\alpha \leq 1)$ is the solution of the equation

$$
\delta=\alpha+\frac{2}{\pi} \tan ^{-1}\left(\frac{\alpha \sin \frac{\pi}{2}(1-t(A, B, c))}{\frac{(c+p)(1-B)+A-1}{1-B}+\alpha \cos \frac{\pi}{2}(1-t(A, B, c))}\right)
$$

when

$$
t(A, B, c)=\frac{2}{\pi} \sin ^{-1}\left(\frac{p(A-B)}{(c+p)\left(1-B^{2}\right)-p(1-A B)}\right)
$$

Proof. Let

$$
q(z)=-\frac{1}{p-\gamma}\left(\frac{z\left(D^{n} F(z)\right)^{\prime}}{D^{n} G(z)}+\gamma\right)
$$

Since $g \in \Sigma_{p}^{*}[n ; A, B]$, from Proposition $2.2, g \in \Sigma_{p}^{*}[n ; A, B]$. 
N. E. CHO AND S. OWA

Using (2.10), we have

$(p-\gamma) q(z) D^{n+p-1} G(z)-(c+p) D^{n+p-1} F(z)=-c D^{n+p-1} f(z)-\gamma D^{n+p-1} G(z)$

Then, by a simple calculation, we get

$$
(p-\gamma)\left(z q^{\prime}(z)+q(z)(-r(z)+c+p)\right)+\gamma(-r(z)+c+p)=-\frac{c z\left(D^{n+p-1} f(z)\right)^{\prime}}{D^{n+p-1} G(z)}
$$

where

$$
r(z)=-\frac{z\left(D^{n+p-1} G(z)\right)^{\prime}}{D^{n+p-1} G(z)}
$$

Hence we have

$$
q(z)+\frac{z q^{\prime}(z)}{-r(z)+c+p}=-\frac{1}{p-\gamma}\left(\frac{z\left(D^{n+p-1} f(z)\right)^{\prime}}{D^{n+p-1} g(z)}+\gamma\right) .
$$

The remaining part of the proof is similar to that of Theorem 2.1 and so we omit it.

Letting $n=-p+1, A=1, B=0$ and $\delta=1$ in Theorem 2.3, we have

Corollary 2.4. Let $c>0$ and $f \in \Sigma$. If

$$
-\operatorname{Re}\left\{\frac{z f^{\prime}(z)}{g(z)}\right\}>\gamma(0 \leq \gamma<p)
$$

for some $g \in \Sigma_{p}$ satisfying the condition

$$
\left|\frac{z g^{\prime}(z)}{g(z)}+p\right|<p
$$

then

$$
-\operatorname{Re}\left\{\frac{z F^{\prime}(z)}{G(z)}\right\}>\gamma
$$

where $F$ and $G$ are given by (2.9) and (2.16), respectively.

Taking $n=0, B \rightarrow A$ and $g(z)=\frac{1}{z^{p}}$ in Theorem 2.3, we have

Corollary 2.5. Let $c>0$ and $f \in \Sigma_{p}$. If 


\section{ARGUMENT ESTIMATES}

$$
\left|\arg \left(-z^{p+1} f^{\prime}(z)-\gamma\right)\right|<\frac{\pi}{2} \delta, \quad(0 \leq \gamma<p ; 0<\delta \leq 1)
$$

then

$$
\left|\arg \left(-z^{p+1} F^{\prime}(z)-\gamma\right)\right|<\frac{\pi}{2} \alpha,
$$

where $F$ is the integral operator given by $(2.9)$ and $\alpha(0<\alpha \leq 1)$ is the solution of the equation

$$
\delta=\alpha+\frac{2}{\pi} \tan ^{-1}\left(\frac{\alpha}{c+p-1}\right)
$$

By using the same methods as in proving Theorem 2.3, we have

Theorem 2.4. Let $f \in \Sigma_{p}$ and choose a positive number $c$ such that

$$
c \geq \frac{1+A}{1+B}-p
$$

where $-1<B<A \leq 1$ and $p \in \mathbb{N}$. If

$$
\left|\arg \left(\frac{z\left(D^{n+p-1} f(z)\right)^{\prime}}{D^{n+p-1} g(z)}+\gamma\right)\right|<\frac{\pi}{2} \delta(\gamma>p ; 0<\delta \leq 1)
$$

for some $g \in \Sigma_{p}^{*}[n ; A, B]$, then

$$
\left|\arg \left(\frac{z\left(D^{n+p-1} F(z)\right)^{\prime}}{D^{n+p-1} G(z)}+\gamma\right)\right|<\frac{\pi}{2} \alpha,
$$

where $F$ and $G$ are given by (2.9) and (2.16), respectively, and $\alpha(0<\alpha \leq 1)$ is the solution of the equation given by (2.17)

Finally, we derive

Theorem 2.5. Let $f \in \Sigma_{p}$. Choose an integer $n$ such that

$$
n \geq \frac{p(1+A)}{1+B}-2 p
$$

where $-1<B<A \leq 1$ and $p \in \mathbb{N}$. If

$$
\left|\arg \left(-\frac{z\left(D^{n+p-1} f(z)\right)^{\prime}}{D^{n+p-1} g(z)}-\gamma\right)\right|<\frac{\pi}{2} \delta(0 \leq \gamma<p ; 0<\delta \leq 1)
$$

for some $g \in \Sigma_{p}^{*}[n ; A, B]$, then 


$$
\left|\arg \left(-\frac{z\left(D^{n+p} F(z)\right)^{\prime}}{D^{n+p} G(z)}-\gamma\right)\right|<\frac{\pi}{2} \delta
$$

where $F$ and $G$ are given by (2.9) and (2.16) with $c=n+p$, respectively.

Proof. From (2.7) and (2.8) with $c=n+p$, we have $D^{n+p-1} f(z)=D^{n+p} F(z)$ Therefore

$$
\frac{z\left(D^{n+p-1} f(z)\right)^{\prime}}{D^{n+p-1} g(z)}=\frac{z\left(D^{n+p} F(z)\right)^{\prime}}{D^{n+p} G(z)}
$$

and the result follows.

Acknowledgement. The author wishes to acknowledge the financial support of the Korea Research Foundation made in the program year of 1997.

\section{References}

1. Nak Eun Cho, On certain subclasses of meromorphically multivalent convex functions, J. Math. Anal. Appl., 193(1995), 255-263.

2. P. Enigenberg,S. S. Miller, P. T. Mocanu and M. O. Reade, On a Briot-Bouquet Differential subordination, General Inequalities, 3(Birkhauser Verlag-Basel), 339-348.

3. M. D. Ganigi and B. A. Uralegaddi, New criteria for meromorphic univalent functions, Bull. Math. Soc. Sci. Math. R. S. Roumanie(N. S.), 33(1989), 9-13.

4. S. S. Miller and P. T. Mocanu,Differential subordinations and univalent functions, Michigan Math. J., 28(1981), 157-171.

5. M. Nunokawa, On the order of strongly starlikeness of strongly convex functions, Proc. Japan Acad. Ser. A Math. Sci., 69(1993), 234-237.

6. H. Silverman and E. M. Silvia, Subclasses of starlike functions subordinate to convex functions, Canad. J. Math., 37(1985), 48-61.

7. B. A. Uralegaddi and H. S. Path, $A$ new criterion for $p$-valent meromorphic convex functions, Math. Chronicle, 18(1989), 75-77.

8. B. A. Uralegaddi and C. Somanatha, $A$ new criterion for meromorphic convex functions, Tamkang J. Math., 19(1988), 43-48.

9. B. A. Uralegaddi and C. Somanatha, Certain subclasses of meromorphic convex functions, Indian J. Math., 32(1990), 49-57.

*Department of Applied Mathematics, Pukyong National University, Pusan 608737, KOREA

**Department of Mathematics, Kinki University, Higashi-Osaka, Osaka 577-8502, JAPAN 